\title{
Finite-size effects and the stabilized spin-polarized jellium model for metal clusters
}

\author{
M. Payami \\ Center for Theoretical Physics and Mathematics, Atomic Energy Organization of Iran, P. O. Box \\ 11365-8486, Tehran, Iran
}

\begin{abstract}
In the framework of spherical geometry for jellium and local spin density approximation, we have obtained the equilibrium $r_{s}$ values, $\bar{r}_{s}(N, \zeta)$, of neutral and singly ionized "generic" $N$-electron clusters for their various spin polarizations, $\zeta$. Our results reveal that $\bar{r}_{s}(N, \zeta)$ as a function of $\zeta$ behaves differently depending on whether $N$ corresponds to a closed-shell or an openshell cluster. That is, for a closed-shell one, $\bar{r}_{s}(N, \zeta)$ is an increasing function of $\zeta$ over the whole range $0 \leq \zeta \leq 1$, and for an open-shell one, it has a decreasing part corresponding to the range $0<\zeta \leq \zeta_{0}$, where $\zeta_{0}$ is a polarization that the cluster assumes in a configuration consistent with Hund's first rule. In the context of the stabilized spin-polarized jellium model, our calculations based on these equilibrium $r_{s}$ values, $\bar{r}_{s}(N, \zeta)$, show that instead of the maximum spin compensation (MSC) rule, Hund's first rule governs the minimum-energy configuration. We therefore conclude that the increasing behavior of the equilibrium $r_{s}$ values over the whole range of $\zeta$ is a necessary condition for obtaining the MSC rule for the minimum-energy configuration; and the only way to end up with an increasing behavior over the whole range of $\zeta$ is to break the spherical geometry of the jellium background. This is the reason why the results based on simple jellium with spheroidal or ellipsoidal geometries show up MSC rule.
\end{abstract}

\section{INTRODUCTION}

Since the production and study of sodium clusters by Knight et al. 11 the physics of metal clusters has attracted much interest. 2.3 Metal clusters are composed of atoms and have properties that are different from both a single atom and the bulk metal. However, when increasing the size of the cluster, its properties evolve to those of the bulk. The many-body technique suitable for these systems is the density functional theory (DFT). 0 U It is a wellknown fact that the properties of alkali metals are dominantly determined by the delocalized valence electrons. In these metals, the pseudopotentials of the ions do not significantly affect the electronic structure, because the Fermi wavelengths of the valence electrons become much larger than the metal lattice constants if one replaces the ions with a uniform positive charge background. This fact allows us to replace the discrete ionic structure by a homogeneous 
positive charge background. This approximation is known as the jellium model (JM). The simplest way of applying the JM to metal clusters is to replace the ions of an $N$-atom cluster by a sphere of uniform positive charge density and radius $R=(z N)^{1 / 3} r_{s}$, where $z$ is the valence of the atom and $r_{s}$ is the bulk value of the Wigner-Seitz radius of the metal. However, since the ionic density near the surface of a metal differs from that of the bulk region, one may resort to the diffuse jellium model (dif-JM) in which the density of the jellium background falls to zero, from the bulk value, within a length of a few atomic sizes. Application of the dif-JM to metal clusters results in a better agreement of the theory and experiment over the JM results. However, in spite of its simplicity and success in predicting some properties of bulk metals and metal clusters, the JM, which was originally developed for bulk metals, has some drawbacks. 1010 In order to overcome the deficiencies of the JM, one should take some details of the ionic structure into account. Among the various methods of improvement, the first attempts that kept the simplicity of the JM and overcame some of the deficiencies of the JM resulted in the development of the stabilized jellium model (SJM) 11 or pseudojellium model.12 13 The SJM was applied to metal clusters 14.10 and improved some results of the simple JM. Montag et al. , 1 in their structure-averaged jellium model (SAJM), which added the ionic surface energy to the SJM energy functional, made it more suitable for metal clusters.

In other approaches, some researchers relax the spherical geometry and use the JM with spheroidal or ellipsoidal shapes, 1719 which are suitable for open-shell clusters. Relaxing the shape of the jellium background as well as its density distribution while keeping charge neutrality at every point in space, called the ultimate jellium model (UJM), was introduced by Koskinen et al.20 Since the bulk density in the UJM $\left(r_{s}=4.18\right)$ is close to that of sodium, its results can be compared with experiments on Na metal clusters. 21.22 However, since the jellium density and the electron density in the UJM are locally equal everywhere, the UJM cannot describe the ionized clusters.

In a recent work, by taking the spin degrees of freedom into account in the process of stabilization, we have generalized the SJM to the stabilized spin-polarized jellium model (SSPJM) ${ }^{23}$ In the SJM, the equilibrium bulk density is a free parameter and the experimental value is used for it. However, in the SSPJM the equilibrium bulk density parameter, $\bar{r}_{s}(\infty, \zeta)$, is polarization dependent and to the best of our knowledge, no experimental data are available for it. Therefore in the SSPJM we take

$$
\bar{r}_{s}^{\mathrm{X}}(\infty, \zeta)=\bar{r}_{s}^{\mathrm{X}}(\infty, 0)+\Delta r_{s}^{\mathrm{EG}}(\infty, \zeta),
$$

in which $\bar{r}_{s}^{\mathrm{X}}(\infty, 0)$ is the equilibrium bulk value for the spin-compensated system which takes the experimental value of metal $\mathrm{X}$, and $\Delta r_{s}^{\mathrm{EG}}(\infty, \zeta)$ is obtained by the application of the local spin-density approximation (LSDA) to the infinite electron-gas system. All equations throughout this paper are expressed in Rydberg atomic units. It turns out that $\Delta r_{s}^{\mathrm{EG}}(\infty, \zeta)$ is an increasing function of $\zeta$. By taking $\zeta=\left(N_{\uparrow}-N_{\downarrow}\right) / N$ for a monovalent metal cluster, and calculating the corresponding $\Delta r_{s}^{\mathrm{EG}}(\infty, \zeta)$, it is possible to determine the appropriate radius for the spherical jellium through $R(N, \zeta)=N^{1 / 3} \bar{r}_{s}(\infty, \zeta)$. Therefore, any variation in $N_{\uparrow}$ and $N_{\downarrow}$, keeping $N=N_{\uparrow}+N_{\downarrow}$ constant, leads to different values for $\zeta$ and thereby, different values for $R(N, \zeta)$.

Application of this model for the energy calculation of metal clusters show that the energy is minimized for a configuration with maximum spin-compensation (MSC) $2^{3}$ That is, for 
clusters with an even number of electrons, $N$, the number of up-spin electrons, $N_{\uparrow}$, equals the number of down-spin electrons, $N_{\downarrow}$; and for an odd number of electrons, $N_{\uparrow}-N_{\downarrow}=1$. This MSC rule leads to the fine structure in $\Delta_{2}(N)$ (see Fig. 5 of Ref. 23) and the odd-even alternations in the ionization energies [Fig. 7(c) of Ref. 23].

In the present paper, keeping the spherical geometry for the jellium, we have investigated the finite-size effects on the equilibrium $r_{s}$ values, and their consequences on the SSPJM calculations. First, using the LSDA, we have found $\bar{r}_{s}(N, \zeta)$, the equilibrium $r_{s}$ values of the closed-shell $N$-electron neutral and singly ionized "generic" clusters with $N=2,8,18,20,34,40$ for all possible polarizations. By generic we mean no specific metal but a simple jellium sphere which can assume its equilibrium size for any given value of $N$. By fitting the calculated results to a polynomial with even powers of $\zeta$ (in the absence of magnetic fields, the physical properties are invariant under the transformation $\zeta \rightarrow-\zeta$ ), we have found two analytic equations for $\Delta r_{s}(\zeta)$, one for neutral and the other for singly ionized clusters. Employing these analytic equations in the SSPJM calculations for $\mathrm{Na}$ clusters shows that as before, the MSC rule is at work and the results do not show any significant changes over our previous results on the ionization energies.23 In the next step, we have found the values $\bar{r}_{s}(N, \zeta)$ for all different neutral and singly ionized generic clusters $(N \leq 42)$ with different spin configurations $(0 \leq \zeta \leq 1)$, and have shown that $\bar{r}_{s}(N, \zeta)$ behaves differently for open-shell and closed-shell clusters. That is, we have found that for a closed-shell cluster (and its two nearest neighbors) it is an increasing function of $\zeta$ over the whole interval $0 \leq \zeta \leq 1$, whereas for an open-shell cluster (except for the two nearest neighbors of a closed-shell cluster), it has a decreasing behavior over $0 \leq \zeta \leq \zeta_{0}$, and an increasing behavior over $\zeta_{0} \leq \zeta \leq 1$. However, in both open-shell and closed-shell clusters, the global minimum of energy, i.e., the ground-state energy, corresponds to a configuration in which $\bar{r}_{s}(N, \zeta)$ is a minimum. Here, $\zeta_{0}$ corresponds to an electronic configuration for which Hund's first rule is satisfied. By subtracting the ground-state energy of a singly ionized generic cluster from that of its neutral counterpart, we obtain the ionization energy of that generic cluster. Calculation of these ionization energies for $N \leq 42$ shows a good agreement with experimental results on Na clusters. We see that, in the ionization-energy plot, although the saw-toothed behavior remains, the pronounced shell effects near closed shells, seen in the simple JM results, are substantially reduced. Thanks to the appreciable reduction of the shell effects in the ionization energy results of the above-mentioned calculations, we have performed the SSPJM calculations using the set of values $\bar{r}_{s}(N, \zeta)$. The results show that, instead of the MSC rule, Hund's first rule is governing the ground-state configuration. We have also performed simple JM-LSDA calculations for the ground-state energies of $\mathrm{Na}$ clusters but, instead of using the ordinary bulk $r_{s}$ value (3.99), we have used the increasing function $\bar{r}_{s}(\infty, \zeta)$ as in Eq. (11). The results show that, here also, Hund's first rule remains at work and therefore, we conclude that with spherical geometries, the increasing behavior of $\Delta r_{s}^{\mathrm{EG}}(\infty, \zeta)$ is a necessary condition (but not sufficient) for realizing the MSC rule, and the energy corrections over the simple JM due to stabilization are also needed. Therefore, in order to improve the SSPJM results one should insist on the increasing behavior for $\bar{r}_{s}(N, \zeta)$. The only way which guarantees such behavior for all values of $N$ is to relax the spherical constraint on the geometry and consider ellipsoidal shapes for open shells. Using the LSDA and ellipsoidal geometry, one could obtain $\bar{r}_{s}(N, \zeta)$, for a given set of values of $N$ and $\zeta$, by finding the values of the ellipsoid axes which correspond to the 
minimum of energy. We expect that in this case $\bar{r}_{s}(N, \zeta)$ will be an increasing function of $\zeta$ for both open-shell and closed-shell clusters. Then, if one performs the SSPJM calculations for ellipsoidal clusters using these new increasing functions $\bar{r}_{s}(N, \zeta)$, one would obtain the MSC rule (and thereby the odd-even alternations) with improved results.

The organization of this paper is as follows. Section $\square$ is devoted to calculational schemes. In Sec. III we present the results of our calculations. In Sec. IV we conclude the work.

\section{CALCULATIONAL SCHEMES}

The energy functional in the SSPJM is given by 23

$$
\begin{aligned}
E_{\mathrm{SSPJM}}\left[n_{\uparrow}, n_{\downarrow}, n_{+}\right]= & E_{\mathrm{JM}}\left[n_{\uparrow}, n_{\downarrow}, n_{+}\right]+\left(\varepsilon_{\mathrm{M}}(\bar{n})+\bar{w}_{R}(\bar{n})\right) \int d \mathbf{r} n_{+}(\mathbf{r}) \\
& +\langle\delta v\rangle_{\mathrm{WS}}(\bar{n}) \int d \mathbf{r} \Theta(\mathbf{r})\left[n(\mathbf{r})-n_{+}(\mathbf{r})\right]
\end{aligned}
$$

in which $E_{\mathrm{JM}}$ is the energy fuctional of the simple JM, $\varepsilon_{\mathrm{M}}$ is the Madelung energy, $\bar{w}_{R}$ is the average value of the repulsive part of the pseudopotential, and $\langle\delta v\rangle_{\mathrm{WS}}$ is the average of the difference potential over the Wigner-Seitz cell and the difference potential, $\delta v$, is defined as the difference between the pseudopotential of a lattice of ions and the electrostatic potential of the jellium background. $n_{\uparrow}(\mathbf{r})$ and $n_{\downarrow}(\mathbf{r})$ are, respectively, the up-spin and down-spin electron densities with the total electron density given by $n(\mathbf{r})=n_{\uparrow}(\mathbf{r})+n_{\downarrow}(\mathbf{r})$; and $\bar{n}$ is the uniform jellium density which is $\zeta$-dependent; for our previous SSPJM calculations we have used the values given by Eq. (四). In the spherical JM, the functions will depend only on the radial variable. The function $\Theta(\mathbf{r})$ becomes a simple radial step function, $\theta(R-r)$, where $R=N^{1 / 3} \bar{r}_{s}$ with $\bar{r}_{s}=(3 / 4 \pi \bar{n})^{1 / 3}$. In the case of jellium with a sharp boundary, $n_{+}(r)=\bar{n} \theta(R-r)$, whereas for the diffuse case we take 8

$$
n_{+}(r)=\left\{\begin{array}{l}
\bar{n}\left\{1-(R+t) e^{-R / t}[\sinh (r / t)] / r\right\}, \quad r \leq R \\
\bar{n}\left\{1-((R+t) / 2 R)\left(1-e^{-2 R / t}\right)\right\} R e^{(R-r) / t} / r, \quad r>R,
\end{array}\right.
$$

where $t$ is a parameter related to the surface thickness.

To evaluate the total energy of a cluster, we solve the Kohn-Sham (KS) equations selfconsistently. The effective potential in the KS equations for the SSPJM calculations is given by

$$
v_{\mathrm{eff}}^{\sigma}\left(\left[n_{\uparrow}, n_{\downarrow}, n_{+}\right] ; \mathbf{r}\right)=\phi\left(\left[n, n_{+}\right] ; \mathbf{r}\right)+v_{x c}^{\sigma}\left(\left[n_{\uparrow}, n_{\downarrow}\right] ; \mathbf{r}\right)+\langle\delta v\rangle_{\mathrm{WS}}(\bar{n}) \Theta(\mathbf{r}),
$$

where

$$
\phi\left(\left[n, n_{+}\right] ; \mathbf{r}\right)=2 \int d \mathbf{r}^{\prime} \frac{\left[n\left(\mathbf{r}^{\prime}\right)-n_{+}\left(\mathbf{r}^{\prime}\right)\right]}{\left|\mathbf{r}-\mathbf{r}^{\prime}\right|} .
$$

which appears in the electrostatic part of the total energy of the simple JM energy functional

$$
\begin{aligned}
E_{\mathrm{JM}}\left[n_{\uparrow}, n_{\downarrow}, n_{+}\right]= & T_{s}\left[n_{\uparrow}, n_{\downarrow}\right]+E_{x c}\left[n_{\uparrow}, n_{\downarrow}\right] \\
& +\frac{1}{2} \int d \mathbf{r} \phi\left(\left[n, n_{+}\right] ; \mathbf{r}\right)\left[n(\mathbf{r})-n_{+}(\mathbf{r})\right],
\end{aligned}
$$


and

$$
v_{x c}^{\sigma}\left(\left[n_{\uparrow}, n_{\downarrow}\right] ; \mathbf{r}\right)=\frac{\delta E_{x c}}{\delta n_{\sigma}(\mathbf{r})}, \quad \sigma=\uparrow, \downarrow .
$$

For $E_{x c}$ we use the LSDA with the Perdew-Wang parametrization for the correlation
part. 4 -

\section{RESULTS AND DISCUSSIONS}

In the first step, by solving the KS equations for spherical geometries of the jellium in the LSDA, and finding the minimum energies, we have obtained $\bar{r}_{s}(N, \zeta)$, the equilibrium $r_{s}$ values of the closed-shell neutral and singly ionized jellium clusters with $N=2,8,18,20,34,40$ electrons. In the calculations we have considered all different possible polarizations. That is, for an $N$-electron cluster $\left(N=N_{\uparrow}+N_{\downarrow}\right)$, we have found $\bar{r}_{s}(N, \zeta)$ for all different polarizations $(0 \leq \zeta \leq 1)$ corresponding to the configurations $N_{\downarrow}=N_{\uparrow}, N_{\downarrow}=N_{\uparrow}-1, N_{\downarrow}=N_{\uparrow}-2$, $\ldots, N_{\downarrow}=0$. The results are shown in Figs. 1(a) and 1(b). We see that for these closed-shell clusters, $\bar{r}_{s}$ is an increasing function of $\zeta$. By a least-square fitting of the results to the polynomial

$$
r_{s}(\zeta)=a_{0}+a_{2} \zeta^{2}+a_{4} \zeta^{4}+a_{6} \zeta^{6}
$$

we have obtained $a_{0}=4.28, a_{2}=2.15, a_{4}=-2.41, a_{6}=1.84$ for neutral, and $a_{0}=4.62$, $a_{2}=1.49, a_{4}=-3.31, a_{6}=3.92$ for singly ionized clusters. In Figs. 11(a) and 1)(b), we have also shown plots using Eq. (8) for the two sets of the coefficients and compared with the bulk function $\bar{r}_{s}^{\mathrm{EG}}(\infty, \zeta)$. The values of $a_{0}$ show that the simple JM with LSDA predicts a larger atomic spacing for a cluster than the bulk value $\left(a_{0}>4.18\right)$. Regardless of the $a_{0}$ values (since we are studying the variations of $r_{s}$ with respect to $\zeta$ ), we have performed our SSPJM calculations using

$$
\Delta r_{s}(\zeta)=a_{2} \zeta^{2}+a_{4} \zeta^{4}+a_{6} \zeta^{6}
$$

with corresponding coefficients for neutral and singly ionized clusters. Figure 2 compares the plots of Eq. (9) for neutral and singly ionized clusters with $\Delta r_{s}^{\mathrm{EG}}(\infty, \zeta)$ of the bulk jellium. We see that they are increasing functions of $\zeta$. Using Eq. (9), we have performed the dif-SSPJM calculations for Na clusters with $\bar{r}_{s}(\infty, 0)=3.99$. After the self-consistent calculations of the KS equations, we have obtained the dif-SSPJM ground-state total energies of the neutral and singly ionized Na clusters $(N \leq 42)$. The calculations show that the MSC rule is governing the ground-state configuration. In the dif-SSPJM calculations, we have taken $t=1$. In Fig. 3 we have plotted the ionization energies and compared them with our previous results 23 and also with the experimental values. As is shown, there are no significant changes over the previous results.

In the second step, using JM-LSDA we have found the values $\bar{r}_{s}(N, \zeta)$ for all different neutral and singly ionized jellium clusters $(N \leq 42)$ with different spin polarizations. Here, for a cluster with specified values of $N$ and $\zeta$, the value $\bar{r}_{s}(N, \zeta)$ minimizes the total energy of the cluster. In Fig. 4 we have shown the values of $\bar{r}_{s}(N, \zeta)$ and corresponding total energies per electron, $\bar{E}(N, \zeta) / N$, as functions of the number of the electrons, $N$, for neutral 
jellium clusters. The two plots show the same structure. That is, the maxima and minima of these two plots correspond to the same values of $N$. The values of the ground-state energies correspond to polarizations $\zeta_{0}$ consistent with Hund's first rule and form the Hund curve. In each of the plots, the uppermost value for a given $N$ corresponds to the configuration with MSC. The values in between correspond to intermediate polarizations. We therefore conclude that for an open-shell cluster the functions $\bar{r}_{s}(N, \zeta)$ and $\bar{E}(N, \zeta) / N$ have minima for a polarization $\zeta_{0} \neq 0$ which is consistent with Hund's first rule.25 28 That is, for openshell clusters (except for the nearest neighbors to the closed-shell cluster) $\bar{r}_{s}$ and $\bar{E}(N, \zeta)$ are decreasing functions of $\zeta$ for $0 \leq \zeta \leq \zeta_{0}$ and increasing functions for $\zeta_{0} \leq \zeta \leq 1$. In the bulk jellium, the function $\bar{r}_{s}^{\mathrm{EG}}(\infty, \zeta)$ is an increasing function over the whole range $0 \leq \zeta \leq 1$. In Fig. 5 we have compared the functions $\bar{r}_{s}(27, \zeta)$ and $\bar{r}_{s}(34, \zeta)$ with $\bar{r}_{s}^{\mathrm{EG}}(\infty, \zeta)$. We see that for the closed-shell cluster $(N=34)$ the volume is an increasing function of $\zeta$ on the whole range $0 \leq \zeta \leq 1$. But for the open-shell cluster $(N=27)$ the volume decreases with increasing $\zeta$ in the range $0<\zeta \leq 7 / 27$ and expands with $\zeta$ in the range $7 / 27 \leq \zeta \leq 1$. The value $\zeta_{0}=7 / 27$ is due to the half-filled shell $(l=3)$ of the up-spin band. Also, we note that $\bar{r}_{s}(27, \zeta)>\bar{r}_{s}^{\mathrm{EG}}(\infty, \zeta)$ over the whole range $0 \leq \zeta \leq 1$. Perdew et al. have also studied the equilibrium size of spherical clusters of stabilized jellium from a different point of view.

Figure 6 shows the ground state $\bar{E} / N$ and its corresponding $\bar{r}_{s}$ for neutral and singly ionized jellium clusters as functions of $N$, the number of electrons. The energies correspond to the equilibrium $r_{s}$ values for configurations consistent with Hund's first rule. Here, $N$ is the number of electrons which for neutral clusters equals the number of the ions, and for singly ionized clusters it is one less than the number of the ions. The ionization energy of an $\mathrm{N}$-atom jellium cluster is given by

$$
I(N)=\bar{E}^{\text {ion }}\left(N-1, \zeta_{0}^{\prime}\right)-\bar{E}^{\text {neut }}\left(N, \zeta_{0}\right),
$$

where $\bar{E}^{\text {neut }}\left(N, \zeta_{0}\right)$ is the ground-state energy of the neutral $N$-electron jellium cluster which occurs at polarization $\zeta_{0}$ consistent with Hund's rule, and $\bar{E}^{\text {ion }}\left(N-1, \zeta_{0}^{\prime}\right)$ is the ground state energy of the singly ionized cluster with $N-1$ electrons and $N$ positive ions. Obviously, the polarizations $\zeta_{0}$ and $\zeta_{0}^{\prime}$ are not the same. In Fig. 0 we have plotted the ionization energies of the jellium clusters $(N \leq 42)$ obtained from Eq. (10), and compared them with the results obtained from the simple JM calculations (with $r_{s}=3.99$ ) and experiment on Na clusters. The relevance of this comparison with $\mathrm{Na}$ results is due to the fact that the equilibrium $r_{s}$ value of the bulk jellium, 4.18, is very close to that of the bulk Na. Except for very small clusters, the results based on the equilibrium $r_{s}$ values show a better agreement with experiment than the results of simple JM calculations for Na clusters.

We saw, in the second step, that if the jellium clusters assume their individual equilibrium volumes, then the ionization energies improve. Therefore, we are naturally led to define a new set of $\Delta r_{s}$ for our SSPJM calculations which are obtained from

$$
\Delta r_{s}(N, \zeta)=\bar{r}_{s}(N, \zeta)-a_{0} .
$$

Here, $a_{0}=4.28$ for neutral and $a_{0}=4.62$ for singly ionized clusters; $\bar{r}_{s}(N, \zeta)$ is the equilibrium $r_{s}$ value for a cluster with given values of $N$ and $\zeta$. Using Eq. (11), we have performed the SSPJM calculations for Na. The results of calculations show that here (in contrast to the MSC rule) the energy of a cluster is minimized for a configuration consistent with Hund's first rule. This argument is valid for both neutral and singly ionized clusters. This behavior 
has its roots in the decreasing behavior of $\bar{r}_{s}(N, \zeta)$ over the range $0<\zeta \leq \zeta_{0}$ for open-shell clusters. We have also checked whether or not taking account of the volume change as a function of polarization, as given by Eq. (1), in the simple JM calculations gives rise to the MSC rule. The results show that Hund's first rule remains at work and therefore we conclude that, in order to obtain MSC configuration for the ground state, not only $\Delta r_{s}$ should be an increasing function of $\zeta$ but one should also include the two corrections, due to the stabilization, in the simple JM energy. That is, one should use the SSPJM energy along with an increasing function for $\Delta r_{s}$. This condition will be met if one relaxes the spherical constraint on the jellium and considers ellipsoidal shapes for open-shell clusters. It is then possible to obtain $\bar{r}_{s}(N, \zeta)$ for a given set of values of $N$ and $\zeta$, by finding the values of the ellipsoid axes that minimize the total energy. We expect that under these conditions $\bar{r}_{s}(N, \zeta)$ becomes an increasing function of $\zeta$ for all clusters. This expectation is due to the fact that addition of a nonsymmetric perturbation to a spherical potential in a single-particle Hamiltonian lifts the orbital degeneracies and each degenerate level splits into $(2 l+1)$ new levels with different energies. Each of these levels will contain at most two electrons with opposite spins. Then, any increment in the polarization is accompanied by a transition of a spin-down electron to an unoccupied level and a successive spin-flip. This is the only way that one can increase the polarization consistent with Pauli's exclusion principle. This process resembles the process of increasing the polarization in a closed-shell spherical cluster which results in increasing its equilibrium $r_{s}$ value. It is a well-known fact that the open-shell clusters lose their spherical geometry due to the Jahn-Teller effect,29 and that is why the MSC rule and the odd-even alternations are observed in experimental data for alkali metal clusters. In short, using a new set of increasing functions $\bar{r}_{s}(N, \zeta)$ - which is obtained using ellipsoidal geometries for simple JM and LSDA- in the SSPJM calculations for ellipsoidal geometries of the jellium will lead to the MSC configuration for the ground state of the cluster. Work in this direction is in progress.

Finally, it should be mentioned that in the context of the SSPJM one could fix, at the beginning, the pseudopotential core radius for a bulk system of a given species with a given fixed polarization; and then use this value in the SSPJM energy functional for a finite cluster (transferability condition on the pseudopotential). 15 Then, one could obtain the equilibrium radius of the jellium by finding that $r_{s}$ value which minimizes the total energy. We have performed such calculations and obtained further agreement to the experimental results, which will appear elsewhere.

\section{CONCLUSION}

In this paper, keeping the spherical geometry, we have considered the finite-size effects on the equilibrium $r_{s}$ values. Our calculations show that for a given $N$-electron cluster, the quantity $\bar{r}_{s}(N, \zeta)$ behaves differently for an open-shell and a closed-shell cluster. That is, this equilibrium $r_{s}$ value is an increasing function of $\zeta$ over the whole range $0 \leq \zeta \leq 1$ for a closed-

shell cluster; whereas for an open-shell cluster it is a decreasing function over $0<\zeta \leq \zeta_{0}$ and an increasing function over $\zeta_{0} \leq \zeta \leq 1$. Here, $\zeta_{0}$ is a polarization corresponding to a configuration consistent with Hund's first rule. Our SSPJM calculations based on equilibrium $r_{s}$ values show that, in contrast to the MSC rule, Hund's first rule is at work. This behavior is due to the fact that $\bar{r}_{s}(N, \zeta)$ has a decreasing part for an open-shell cluster. 
We therefore conclude that to realize the MSC rule in the SSPJM calculations with $\bar{r}_{s}(N, \zeta)$, and thereby the odd-even alternation, one should lift the spherical constraint on the jellium and let it assume ellipsoidal shapes.

\section{ACKNOWLEDGMENTS}

The author would like to thank John P. Perdew for reading the manuscript and his helpful discussions on the subject. He also thanks N. Nafari for his useful discussions. 


\section{REFERENCES}

${ }^{1}$ W. D. Knight, K. Clemenger, W. A. de Heer, W. A. Saunders, M. Y. Chou, and M. L. Cohen, Phys. Rev. Lett. 52, 2141 (1984).

${ }^{2}$ W. A. de Heer, Rev. Mod. Phys. 65, 611 (1993), and references therein.

${ }^{3}$ M. Brack, Rev. Mod. Phys. 65, 677 (1993), and references therein.

${ }^{4}$ P. Hohenberg and W. Kohn, Phys. Rev. 136, B864 (1964).

${ }^{5}$ W. Kohn and L. J. Sham, Phys. Rev. 140, A1133 (1965).

${ }^{6}$ R. G. Parr and W. Yang, Density Functional Theory of Atoms and Molecules (Oxford University Press, New York 1989), and references therein.

${ }^{7}$ R. M. Dreizler and E. K. U. Gross, Density Functional Theory (Springer, Berlin, 1990), and references therein; Density Functional Theory, edited by E. K. U. Gross and R. M. Dreizler (Plenum, New York, 1995).

${ }^{8}$ A. Rubio, L. C. Balbás, and J. A. Alonso, Z. Phys. D 19, 93 (1991).

${ }^{9}$ N. D. Lang and W. Kohn, Phys. Rev. B 1, 4555 (1970).

${ }^{10}$ N. W. Ashcroft and D. C. Langreth, Phys. Rev. 155, 682 (1967).

11 J. P. Perdew, H. Q. Tran, and E. D. Smith, Phys. Rev. B 42, 11627 (1990).

12 C. A. Utreras-Diaz and H. B. Shore, Phys. Rev. B 40, 10345 (1989).

13 J. H. Rose and H. B. Shore, Phys. Rev. B 43, 11605 (1991).

${ }^{14}$ M. Brajczewska, C. Fiolhais, and J. P. Perdew, Int. J. Quantum Chem., Quantum Chem. Symp. 27, 249 (1993).

15 J. P. Perdew, M. Brajczewska, and C. Fiolhais, Solid State Commun. 88, 795 (1993).

${ }^{16}$ B. Montag, P. -G. Reinhard, and J. Meyer, Z. Phys. D 32, 125 (1994).

17 W. Ekardt and Z. Penzar, Phys. Rev. B 43, 1322 (1991).

${ }^{18}$ T. Hirschmann, M. Brack, and J. M. Meyer, Ann. Phys. (Leipzig) 3, 336 (1994).

${ }^{19}$ C. Yannouleas and Uzi Landman, Phys. Rev. B 51, 1902 (1995).

${ }^{20}$ M. Koskinen, P. O. Lipas, M. Manninen, Z. Phys. D 35, 285 (1995).

${ }^{21}$ M. M. Kappes, M. Schär, U. Röthlisberger, C. Yeretzian, and E. Schumacher, Chem. Phys. Lett. 143, 251 (1988).

${ }^{22}$ M. L. Homer, J. L. Persson, E. C. Honea, and R. L. Whetten, Z. Phys. D 22, 441 (1991).

${ }^{23}$ M. Payami and N. Nafari, J. Chem. Phys. 109, 5730 (1998).

24 J. P. Perdew and Y. Wang, Phys. Rev. B 45, 13244 (1992).

${ }^{25}$ J. C. Slater, Quantum Theory of Atomic Structure (McGraw-Hill, New York, 1960), Vol. 2.

${ }^{26}$ H. Tatewaki and K. Tanaka, J. Chem. Phys. 60, 601 (1974).

${ }^{27}$ H. Tatewaki, T. Koga, Y. Sakai, and A. J. Thakkar, J. Chem. Phys. 101, 4945 (1994).

28 T. Koga, H. Aoki, and H. Tatewaki, Theor. Chim. Acta 92, 281 (1995).

${ }^{29}$ H. A. Jahn and E. Teller, Proc. R. Soc. London, Ser. A 161, 220 (1937). 


\section{FIGURES}

FIG. 1. The equilibrium $r_{s}$ values in atomic units as functions of the polarization $\zeta$. Large squares, diamonds, crosses, pluses, small squares, triangles correspond to clusters with $N=2,8,18$, 20, 34, 40 electrons, respectively, for (a) neutral, and (b) singly ionized closed-shell clusters. The solid lines and the dashed lines correspond to fitted and bulk values, respectively.

FIG. 2. The increments of $r_{s}$ in atomic units as functions of $\zeta$. The dotted and dashed lines correspond to the fitted result [ see Eq. (9) ] for neutral and singly ionized clusters, respectively. The solid line corresponds to the bulk jellium.

FIG. 3. The ionization energies as functions of the number of atoms for Na clusters. The diamonds correspond to the dif-SSPJM results obtained using Eq. (11) and the triangles correspond to the dif-SSPJM results obtained using Eq. (9). The small squares are experimental results.

FIG. 4. The equilibrium $r_{s}$ values in atomic units and their corresponding total energies per electron in electron volts as functions of the number of electrons for neutral clusters with different spin configurations. The triangles correspond to the configurations consistent with Hund's first rule, and the uppermost symbols for each $N$ correspond to the MSC configurations. The symbols between the Hund and the MSC configurations correspond to intermediate polarizations. The horizontal dashed line below the $\bar{r}_{s}$ curve corresponds to the value $r_{s}=4.18$ of the bulk jellium.

FIG. 5. The equilibrium $r_{s}$ values in atomic units as functions of $\zeta$. The solid squares and diamonds correspond to the closed-shell $(N=34)$ and open-shell $(N=27)$ clusters, respectively. The dashed line corresponds to the bulk which is obtained using Eq. (17) of Ref. 23. As is seen, the equilibrium $r_{s}$ of the open-shell cluster has a decreasing part before $\zeta_{0}=7 / 27$, whereas for the closed-shell cluster it is an increasing function over the whole range $0 \leq \zeta \leq 1$.

FIG. 6. The equilibrium $r_{s}$ values in atomic units and their corresponding total energies per electron in electron volts for the ground-state configurations (i.e., the configurations consistent with Hund's first rule) of $N$-electron clusters. The solid squares correspond to neutral and the diamonds correspond to singly ionized $N$-electron clusters. As is expected, for small clusters the differences are high and for large clusters these differences approach zero.

FIG. 7. The ionization energies in electron volts. The diamonds correspond to the simple JM results for $\mathrm{Na}$ using the bulk value $r_{s}=3.99$. The small solid squares correspond to the ionization energies of jellium clusters in their equilibrium states obtained using Eq. (10). It shows a good agreement with the experimental values of $\mathrm{Na}$, which are shown as the large squares. 